\title{
ACCURATE KAP METER CALIBRATION AS A PREREQUISITE FOR OPTIMISATION IN PROJECTION RADIOGRAPHY
}

\author{
Alexandr Malusek, Michael Sandborg and Gudrun Alm Carlsson
}

\section{Linköping University Post Print}

\section{Tweet}

N.B.: When citing this work, cite the original article.

Original Publication:

Alexandr Malusek, Michael Sandborg and Gudrun Alm Carlsson, ACCURATE KAP METER CALIBRATION AS A PREREQUISITE FOR OPTIMISATION IN PROJECTION RADIOGRAPHY, 2016, Radiation Protection Dosimetry, 1-7.

http://dx.doi.org/10.1093/rpd/ncv524

Copyright: Oxford University Press (OUP): Policy B - Oxford Open Option A http://www.oxfordjournals.org/

Postprint available at: Linköping University Electronic Press

http://urn.kb.se/resolve?urn=urn:nbn:se:liu:diva-124662 


\section{Accurate KAP meter calibration as a prerequisite for optimization in projection radiography}

A. Malusek, M. Sandborg and G. Alm Carlsson

Medical Radiation Physics,

Department of Medical and Health Sciences and

Center for Medical Image Science and Visualisation,

Linköping University,

SE 58185 Linköping, Sweden

Corresponding author:

alexandr.malusek@liu.se

fax +46101032895

phone +46101033059

Short running title (max 40 characters): Accurate KAP meter calibration methods 
Accurate KAP meter calibration as a prerequisite for optimization in projection radiography

A. Malusek, M. Sandborg and G. Alm Carlsson

\section{ABSTRACT}

Modern x-ray units register the air kerma area product, $P_{K A}$, with a built-in KAP meter function. Some KAP meters show an energy-dependent bias comparable to the maximum uncertainty articulated by the IEC (25\%), adversely affecting dose-optimisation processes. To correct for the bias, a reference KAP meter calibrated at a standards laboratory and two calibration methods described here can be used to achieve an uncertainty of less than $7 \%$ as recommended by IAEA. A computational model of the reference KAP meter is used to calculate beam quality correction factors for transfer of the calibration coefficient at the standards laboratory, $Q_{0}$, to any beam quality, $Q$, in the clinic. Alternatively, beam quality corrections are measured with an energy independent dosemeter via a reference beam quality in the clinic, $Q_{1}$, to beam quality, $Q$. Biases up to $35 \%$ of built-in KAP meter readings were noted. Energy dependent calibration factors are needed for unbiased $P_{K A}$. 


\section{INTRODUCTION}

The air kerma area product, $P_{K A}$, is a measure related to patient absorbed doses. Its averages for a representative patient population in common diagnostic $\mathrm{x}$-ray procedures for adult patients in individual x-ray rooms are regularly evaluated and compared to national diagnostic reference levels (DRL). Modern x-ray units are equipped with built-in kerma-area product (KAP) meters, which are either transmission ionization chambers or software programs using settings of tube voltage, tube load, filtration and collimators as inputs. Most commonly, a transmission ionization chamber is built into the collimator assembly by the vendor. Such chambers, using conducting layers of indium oxide to provide light transparency, have a high energy dependence.

Optimisation attempts to reduce patient doses by $10-15 \%$ are typically regarded worthwhile provided the clinical image quality is maintained at an acceptable level. Methods to obtain KAP meter readings with higher accuracy than the $25 \%$ (coverage factor $\mathrm{k}=2$ ) accepted in the IEC standard ${ }^{(1)}$ are therefore required in order to make these optimisation attempts meaningful. In fact the $\mathrm{IAEA}^{(2)}$ and ICRU ${ }^{(3)}$ both recommended that KAP meter readings be registered with an accuracy better than $7 \%(\mathrm{k}=2)$. With contemporary KAP meters this can be challenging as the energy dependence of some KAP meters may be large and need to be corrected for.

The tandem calibration method ${ }^{(4)}$ uses a reference KAP meter that is calibrated using a reference beam quality $Q_{0}$ at a standards laboratory. In this method, the reference KAP meter is positioned downstream of the built-in KAP meter. To avoid registering stray radiation from the collimator assembly and yet cover the whole nominal beam area the recommended 
collimator - reference KAP meter distance is about $30 \mathrm{~cm}^{(4,5)}$, but if the beam area is sufficiently small, e.g. $10 \mathrm{~cm} \times 10 \mathrm{~cm}$, the reference KAP meter can be placed for instance 20 $\mathrm{cm}$ above the patient table as in the IAEA recommendation ${ }^{(2)}$. The reference KAP meter needs to be calibrated to measure incident $P_{K A}$, i.e., the air kerma-area product at the position of the reference KAP meter when the reference KAP meter is not present. Using this method, $P_{K A}$ is measured according to its definition, i.e., the inhomogeneities in the distribution of air kerma over the field area (caused for instance by the heel effect) are automatically accounted for. However, applying a single calibration coefficient obtained for the reference beam quality $Q_{0}$ at the standards laboratory for all clinically used beam qualities, $Q$, may lead to biased $P_{K A}$. An alternative to the tandem calibration method is the beam-area method ${ }^{(6)}$, where $P_{K A}$ is estimated as a product of the air kerma, $K$, measured at the reference plane on the beam axis and the beam area, $A$, determined as the area with at least one half of the maximum beam intensity. To reduce the amount of backscattered radiation, the reference plane should be 20 cm above the patient table ${ }^{(2)}$.

Modern radiography and fluoroscopy units use a large range of tube voltages $(40-150 \mathrm{kV})$ and added filtrations and, particularly, heavily copper-filtered beams (0.1-0.9 mm Cu). Many standards laboratories currently cannot provide calibration coefficients for such heavily filtered beams and thus cannot provide calibration coefficients corresponding to all beam qualities Q used in the clinic. Therefore methods to transfer the calibration coefficient derived at the standards laboratory for a specified reference beam quality $Q_{0}$ to beam qualities $Q$ used in the clinic are needed to improve the accuracy of the reported $P_{K A}$.

The aim of this work is to describe two calibration methods that account for the transfer of the calibration coefficient from the standards laboratory beam quality $Q_{0}$ to the clinically used beam qualities $Q$. Both methods use Monte Carlo calculated beam quality corrections factors 
and one method uses in addition an energy independent dosemeter measuring air kerma. Uncertainties in both methods are discussed to explore if the recommended accuracy in $P_{K A}$ of $7 \%(\mathrm{k}=2)$ can be met.

\section{MATERIAL AND METHODS}

\section{Calibration using a reference KAP meter}

The air kerma area product $P_{K A, Q}$ measured by the built-in KAP meter is given as

$$
P_{K A, Q}=N_{P_{K A, Q}} M_{Q}
$$

where $M_{Q}$ is the signal of the built-in KAP meter at a beam quality $Q$, and $N_{P_{K A, Q}}$ is the (true) calibration coefficient. The built-in KAP meter for measurement of $P_{K A, Q}$ in the clinical examinations is typically firmly mounted in the collimator assembly and cannot easily be calibrated at the standards laboratory. In practice, the calibration coefficient is determined by the vendors using the in-situ beam-area calibration method for a limited set of beam qualities only. This limits the accuracy of the calibration coefficient for other beam qualities owing to the energy dependence of the KAP meter. Medical physicists may need to correct such calibration coefficients to obtain accurate $P_{K A, Q}$ for x-ray imaging optimization.

This work proposes two calibration methods based on the tandem calibration: (i) a purely computational method for calculation of beam quality correction factors from beam quality $Q_{0}$ to beam qualities $Q$ (in the following called the computational method) and (ii) calculation of the beam quality correction factor from beam quality $Q_{0}$ to a reference beam quality $Q_{1}$ in the clinic followed by experimental determination of the beam quality correction factor from $Q_{1}$ to $Q$ using an energy independent dosemeter for example an ionization chamber (in the following called the combined method). In both cases, the reference KAP meter is mounted 
downstream of the x-ray collimator assembly and is calibrated to measure incident $P_{K A}$ at a standards laboratory to ensure traceability to a primary standard. In this work, the calibration was done at the secondary standards laboratory of the Swedish Radiation Safety Authority (SSM).

\section{Computational method}

The $P_{K A, Q}$ measured by the reference KAP meter is derived by

$$
P_{K A, Q}=k_{Q, Q_{0}}^{m o d} N_{P_{K A}, Q_{0}}^{r e f} M_{Q}^{r e f}
$$

where $N_{P_{K A}, Q_{0}}^{r e f}$ is the calibration coefficient from the standards laboratory, $M_{Q}^{r e f}$ the signal from the reference KAP meter in the clinical beam $Q$, and $k_{Q, Q_{0}}^{\text {mod }}$ the beam quality correction factor predicted by the computational model ${ }^{(7)}$ of the reference KAP meter. The latter is computed by

$$
k_{Q, Q_{0}}^{\bmod }=\frac{N_{P_{K A}, Q}^{\bmod }}{N_{P_{K A}, Q_{0}}^{m o d}},
$$

where $N_{P_{K A}, \mathrm{Q}}^{\bmod }$ and $N_{P_{K A}, Q_{0}}^{\bmod }$ are the calibration coefficients for the clinical beam quality $Q$ and the reference beam quality $Q_{0}$ at the standards laboratory, respectively, computed using Monte Carlo simulations and x-ray spectra derived from the computational program SpekCalc ${ }^{(8)}$. The computational method is illustrated in figure 1(a). 
(a)

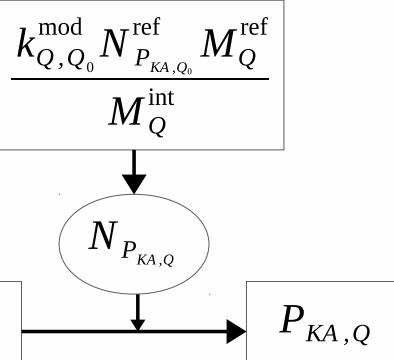

(b)

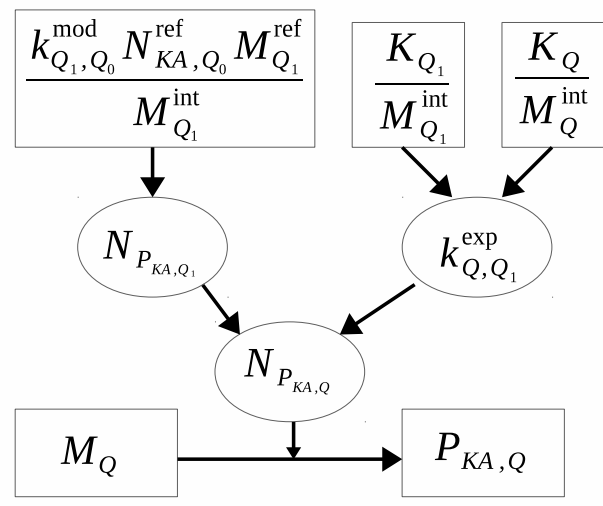

Figure 1. Determination of the air kerma-area product $P_{K A, Q}$ from the reading $M_{Q}$ of the builtin KAP meter. In the computational method (a), the calibration coefficient $N_{P_{K A, Q}}$ of the builin KAP meter is determined using the reference KAP meter. In the combined method (b), the calibration coefficient $N_{P_{K A, Q}}$ of the built-in KAP meter is determined using the reference KAP meter and a dosemeter for air kerma $K_{Q}$ on the beam axis. Beam quality $Q$ is any quality used for patient exposures in the clinic, $Q_{1}$ is the reference beam quality at the clinic and $Q_{0}$ is the reference beam quality at the standards laboratory.

\section{Combined method}

The $P_{K A, Q}$ measured by the reference KAP meter is derived by

$$
P_{K A, Q}=k_{Q, Q_{1}}^{e x p} k_{Q_{1}, Q_{0}}^{\text {mod }} N_{P_{K A}, Q_{0}}^{r e f} M_{Q}^{r e f},
$$

where $k_{Q_{1}, Q_{0}}^{\text {mod }}$ is the beam quality correction factor for the transfer of the calibration coefficient from the standards laboratory to the reference beam quality $Q_{1}$ in the clinic as derived from the computational model, $k_{Q, Q_{1}}^{\text {exp }}$ is the beam quality correction factor for transfer of the calibration coefficient from beam quality $Q_{1}$ to beam quality $Q$ determined experimentally with an energy independent ionization chamber, and $M_{Q}^{r e f}$ and $N_{P_{K A}, Q_{0}}^{r e f}$ have the same 
meaning as in equation (3). The beam quality correction factor $k_{Q, Q_{1}}^{\text {exp }}$ can be determined experimentally using

$$
k_{Q, Q_{1}}^{e x p}=\frac{K_{Q} / M_{Q}^{r e f}}{K_{Q_{1}} / M_{Q_{1}}^{r e f}},
$$

where $K_{Q}$ is air kerma measured on the central beam axis with a small, energy independent dosemeter and $M_{Q}^{r e f}$ is the corresponding signal measured with the reference KAP meter for the beam quality $Q$. Quantities $K_{Q_{1}}$ and $M_{Q_{1}}^{r e f}$ have the same meaning for the reference beam quality $Q_{1}$ at the clinic. Equation (5) follows from the approximation used in the beam-area calibration method, i.e., the integral $\int K d A$ is estimated with the product $K A$, where $K$ is air kerma and $A$ is the cross-sectional area of the beam. As the beam area $A$ is the same for both beam qualities $Q$ and $Q_{l}$ (the collimator settings are the same), the ratio $K_{Q} A /\left(K_{Q_{1}} A\right)$ then simplifies to $K_{Q} / K_{Q_{1}}$. Note that beam quality correction factor $k_{Q, Q_{1}}^{\text {exp }}$ for the built-in KAP meter can be derived similarly by replacing the signal of reference KAP meter in equation (5) with the signal of the built-in KAP meter. The combined method is illustrated in figure 1(b).

If the clinical reference beam quality $Q_{1}$ is selected to be similar to the reference beam quality at the standards laboratory $Q_{0}$, for example RQR5 $(70 \mathrm{kV}, \mathrm{HVL}=2.54 \mathrm{~mm} \mathrm{Al})$, then the beam quality correction factor $k_{Q_{1}, Q_{0}}^{\text {mod }}$ is close to one. The uncertainty in $k_{Q_{1}, Q_{0}}^{\text {mod }}$ is determined by the uncertainties in the computational model of the reference KAP meter and the model used for calculating the $\mathrm{x}$-ray spectra.

\section{Relative error of the built-in KAP meter}


Relative error of the built-in KAP meter was calculated from its reading $M_{Q}^{\text {int }}$ and the $P_{K A, Q}$ provided by the reference KAP meter as

$$
\delta=\frac{M_{Q}^{i n t}-P_{K A, Q}}{P_{K A, Q}}=\frac{M_{Q}^{i n t}}{P_{K A, Q}}-1
$$

Note that equation (6) can be written as

$$
\delta=\frac{M_{Q}^{i n t} / M_{Q}^{i n t}-P_{K A, Q} / M_{Q}^{i n t}}{P_{K A, Q} / M_{Q}^{i n t}}=\frac{N_{P_{K A, Q}}^{i n t}-N_{P_{K A, Q}}}{N_{P_{K A, Q}}}
$$

where $N_{P_{K A, Q}}^{\text {int }}=1$ and $N_{P_{K A, Q}}$ are the calibration coefficients of the built-in and ideal KAP meters, respectively, i.e., the quantity $\delta$ also gives the relative error of the calibration coefficient of the built-in KAP meter.

\section{Computational model of the Vacutec 70157 KAP meter}

The Monte Carlo code PENELOPE ${ }^{(9)}$ was used to simulate energy imparted to the air cavities of the Vacutec model 70157 reference KAP meter for mono-energetic photons emitted from a point source (for details of the model see Malusek et $a l^{(7)}$ ). In the second step, energy imparted to the sensitive volumes was calculated by analytical integration over an energy spectrum of photons, where contributions from individual photon energies were weighted by the number of photons in corresponding energy channels of the spectrum. Clinical energy spectra were obtained using the SpekCalc software ${ }^{(8)}$ for the anode angle of 15 degree, no added filtration, and tube voltages from 40 to $150 \mathrm{kV}$. The spectra were analytically filtered with an Al slab so that the simulated spectrum had the same half value layer, HVL, as that measured at the clinic, see the next section. Clinical energy spectra for $\mathrm{Cu}$-filtered beams were analytically calculated 
using the appropriate Cu-thickness. Computation of energy spectra at SSM is described in Malusek et $a l^{(7)}$.

As shown in Figure 2(a), the beam quality correction factors depend on the indium oxide layer thickness, which the manufacturer treats as proprietary information. To work around this limitation, three thicknesses were simulated $(10,15$ and $20 \mathrm{~nm})$ to investigate the sensitivity of the response to the thickness of this layer. The response between individual KAP meters of the same model results in relatively large variations in the calibration coefficients $N_{P_{K A, Q}}$ (not shown). However, the beam quality correction factors, which are ratios of two calibration coefficients (see equation 3), vary only little between the individual KAP meters ${ }^{(7)}$.

\section{Measurement on clinical imaging equipment}

Measurements were conducted on a clinical radiography system (Siemens Axiom Aristos FX) and a fluoroscopy system (Siemens Axiom Artis Zee MP). The radiography system uses a KAP meter from KERMAX, Scanditronix Wellhöfer $\mathrm{GmbH}$ and allows tube voltages between 40 and $150 \mathrm{kV}$. The fluoroscopy system uses a DIAMENTOR KAP meter (PTW Freiburg, Germany) and tube voltages between 50 and $120 \mathrm{kV}$. The radiography system was operated in clinical mode and $\mathrm{Cu}$ filter thicknesses of $0-0.3 \mathrm{~mm}$ in steps of $0.1 \mathrm{~mm}$ were selected manually. The fluoroscopy system was operated in service mode in order to be able to manually select the added 0.6 or $0.9 \mathrm{~mm} \mathrm{Cu}$ filters. (In clinical mode the Siemens CAREfilter ${ }^{(10)}$ selects the appropriate $\mathrm{Cu}$ filter thickness automatically that is impractical in calibration measurements). The geometry was chosen to minimise the influence of scatter and a lateral beam direction was used with the fluoroscopy unit to move the table couch out of the beam. (Hence the table couch and mattress were not part of the model in this work and 
methods to correct for their attenuation need to be applied separately.) The Vacutec model 70157 reference KAP meter measured the air kerma-area product, $P_{K A, Q}$ and $P_{K A, Q_{1}}$; it was connected to the Barracuda electrometer from RTI Electronics, Sweden. The spherical $3.6 \mathrm{~cm}^{3}$ Exradin A3 ionization chamber, Standard Imaging, Inc., measured the air kerma, $K_{Q}$ and $K_{Q_{1}}$, at the central beam axis; it was connected to a Unidos electrometer from PTW Freiburg, Germany. The HVL of clinical beams was measured with the Barracuda multipurpose detector (MPD), RTI Electronics.

\section{RESULTS}

\section{Beam quality correction factors}

Table 1 shows the computed beam quality correction factors $k_{Q_{1}, Q_{0}}^{\text {mod }}$ for the two clinical x-ray units. The reference beam quality $Q_{1}$ was selected differently for the two clinical units to demonstrate the effect of this choice on the resulting beam quality correction factor and associated uncertainty. When $Q_{1}(70 \mathrm{kV}, 2.8 \mathrm{~mm} \mathrm{Al}, 0.0 \mathrm{~mm} \mathrm{Cu})$ was similar to $Q_{0}, k_{Q_{1}, Q_{0}}^{\text {mod }}$ was close to unity (Aristos FX), but in this case not exactly unity since the x-ray tubes at the two sites had different anode angles and construction materials. When $Q_{1}(70 \mathrm{kV}, 3.0 \mathrm{~mm} \mathrm{Al}$, $0.6 \mathrm{~mm} \mathrm{Cu}$ ) was different from $Q_{0}$, as with Artis Zee MP, $k_{Q_{1}, Q_{0}}^{m o d}$ deviated considerably from unity. In this case it was mainly due to the added $\mathrm{Cu}$ filtration in $Q_{1}$ and to a smaller extent due to the difference in the anode angles between the clinical and standards laboratory systems, which changed the effective photon energy of the beam.

Table 1. Computed beam quality correction factors $k_{Q_{1}, Q_{0}}^{\text {mod }}$ for the two clinical x-ray units and thicknesses of the conductive coating layer of 10, 15 and $20 \mathrm{~nm}$. The reference beam quality at the standards laboratory $Q_{0}$ was RQR5 $(70 \mathrm{kV}, 2.77 \mathrm{~mm} \mathrm{Al}$ filtration) and the reference 
beam quality $Q_{1}$ in the clinic is given in the table. Note that if $Q_{0} \neq Q_{1}$ then $k_{Q_{1}}^{\text {mod }} Q_{0}$ will differ from unity as with the Artis Zee MP unit.

\begin{tabular}{|l|l|l|l|l|}
\hline X-ray unit & $Q_{1}$ & $k_{Q_{1}, Q_{0}}^{\text {mod }}$ & $k_{Q_{1}, Q_{0}}^{\text {mod }}$ & $k_{Q_{1}, Q_{0}}^{\text {mod }}$ \\
& & $10 \mathrm{~nm}$ & $15 \mathrm{~nm}$ & $20 \mathrm{~nm}$ \\
\hline Siemens Axiom Aristos FX & $70 \mathrm{kV}, 2.8 \mathrm{~mm} \mathrm{Al}, 0.0 \mathrm{~mm} \mathrm{Cu}$ & 0.9926 & 0.9906 & 0.9893 \\
\hline Siemens Axiom Artis Zee & $70 \mathrm{kV}, 3.0 \mathrm{~mm} \mathrm{Al}, 0.6 \mathrm{~mm} \mathrm{Cu}$ & 0.87 & 0.84 & 0.82 \\
\hline MP & & & & \\
\hline
\end{tabular}

Figure 2 shows the comparison between the measured $k_{Q, Q_{1}}^{\text {exp }}$ and calculated $k_{Q, Q_{1}}^{\text {mod }}$ for the two clinical units. For some beam qualities, the indium oxide layer thickness of $10 \mathrm{~nm}$ better approximated the measured data than the thickness of $15 \mathrm{~nm}$. The dependence of the beam quality correction factor with tube voltage for the different added filtrations was well described with the model and extended from 0.85 to 1.05 and from 0.95 to 1.30 for the radiography and fluoroscopy systems, respectively. 


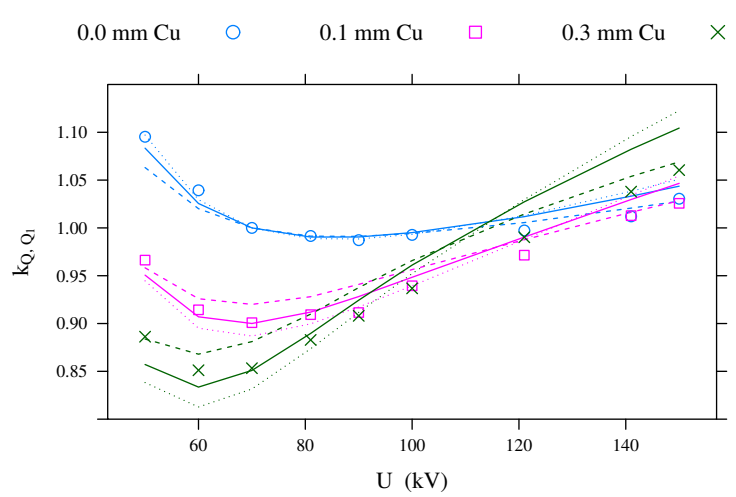

(a)

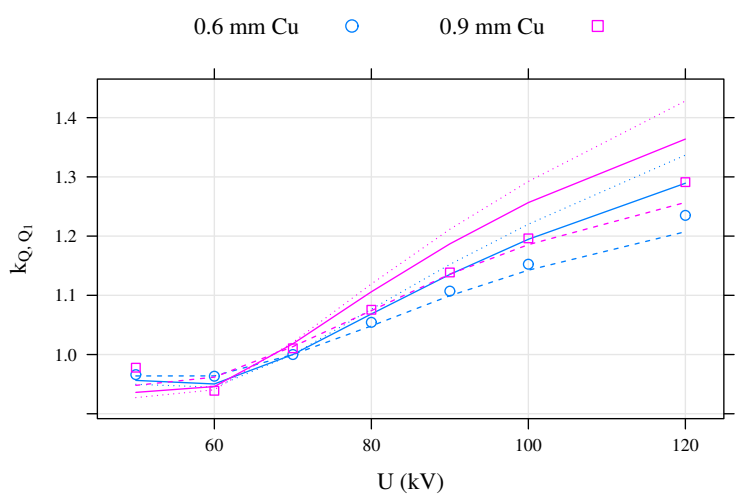

(b)

Figure 2. Comparison between the measured (markers) $k_{Q, Q_{1}}^{e x p}$ and calculated (lines) beam quality correction factor $k_{Q, Q_{1}}^{\text {mod }}$ for the two x-ray units as in Table 1. (a) Siemens Axiom Aristos FX and (b) Siemens Axiom Artis Zee MP. Indium oxide conductive coating layer of $10 \mathrm{~nm}$ (dashed line), $15 \mathrm{~nm}$ (solid line) and $20 \mathrm{~nm}$ (dotted line). Different added filtrations are indicated in the figures. Relative uncertainty $(\mathrm{k}=2)$ in measured values was less than approximately $2 \%$.

\section{Biases of the built-in clinical KAP meter}

Figure 3(a) shows the relative error $\delta$ (or bias) of the $P_{K A}$ reported by the built-in KAP meter as a function of tube voltage, $U$, for added filtrations of $0.0,0.1$, and $0.3 \mathrm{~mm} \mathrm{Cu}$ for the radiography system. The uncertainty in $\delta$ was mainly affected by the uncertainty in $P_{K A}$ measured by the reference KAP meter, but in some cases the limited number of displayed digits of the built-in KAP meter reading notably affected the uncertainty in $\delta$. The latter factor was most likely responsible for the small irregularities in the shape of curves in Figure 3(a). Uncertainties associated with the computational model had small effect on the resulting uncertainty, for instance the curves for the thickness of 10, 15 and $20 \mathrm{~nm}$ almost overlap. This 
was caused by the fact that the beam qualities $Q$ and $Q_{1}$ were similar; the beam quality correction factor $k_{Q_{1}, Q_{0}}^{\text {mod }}$ was close to unity in this case. Note that the resulting biases for the added filtration of $0.3 \mathrm{~mm} \mathrm{Cu}$ and tube voltages above $120 \mathrm{kV}$, exceeded the limit of $25 \%$ defined by $\operatorname{IEC}^{(1)}$.

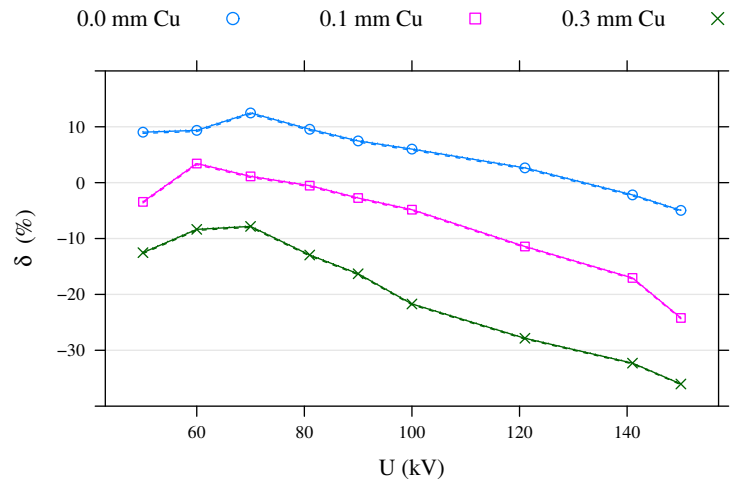

(a)

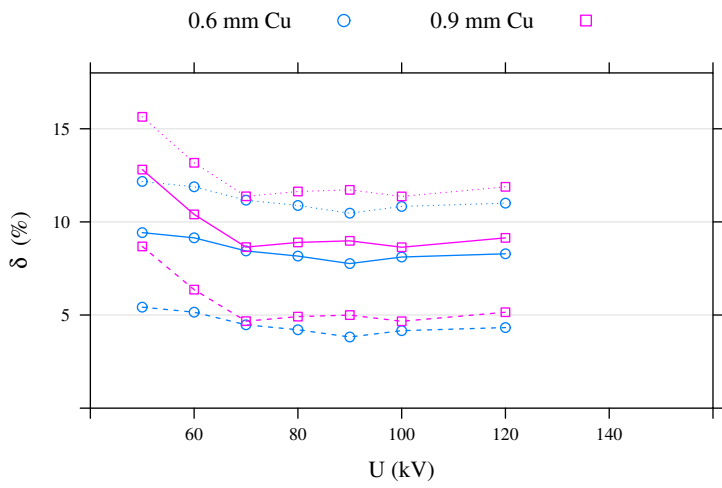

(b)

Figure 3. Relative error $\delta$ of the $\mathrm{P}_{\mathrm{KA}}$ reported by the built-in KAP meter as a function of tube voltage $U$ for (a) Aristos FX and (b) Artis Zee MP determined using the combined method. Curves for the indium oxide conductive coating layer of $10 \mathrm{~nm}$ (dashed line), $15 \mathrm{~nm}$ (solid line) and $20 \mathrm{~nm}$ (dotted line) almost overlap for the Aristos FX unit as the beam quality correction factor $k_{Q_{1}, Q_{0}}^{\text {mod }}$ is close to 1 in this case. The biases for Artis Zee MP are smaller but the uncertainties in $\delta$ are larger than for Aristos FX, here expressed by the variation in $\delta$ due to indium oxide conductive coating layer thickness used in the model to compute $k_{Q_{1}, Q_{0}}^{\text {mod }}$. Different added filtrations are indicated in the figures. Relative uncertainty $(k=2)$ was lower than $5.3 \%$. 
Figure 3(b) shows the relative error $\delta$ of $P_{K A}$ reported by the built-in KAP meter as a function of tube voltage, $U$, for filtrations of 0.6 , and $0.9 \mathrm{~mm} \mathrm{Cu}$ and the fluoroscopy system. The error was smaller compared to the radiography system (Figure 3a) and shows less variation with tube voltage and filtration. However the precision of $\delta$ was less than in Figure 3a since there was a larger uncertainty in the computation of $k_{Q_{1}, Q_{0}}^{\bmod }$ if $Q_{1} \neq Q_{0}$. The indium oxide layer thickness that best fitted data in Figure 2(b) was between 10 and $15 \mathrm{~nm}$. For such thickness, the relative error of the $P_{K A}$ was estimated between 4 and $9 \%$ at $70-120 \mathrm{kV}$ for both $\mathrm{Cu}$ filtrations. As the best fit in Figure 2(b) was closer to $10 \mathrm{~nm}$ than $15 \mathrm{~nm}$, the relative error was most likely less than $6 \%$ for this beam quality. For $0.9 \mathrm{~mm} \mathrm{Cu}$ filtration and tube voltages lower than or equal to $60 \mathrm{kV}$, the uncertainty in the estimated error was larger due to low signal in the Exradin ionization chamber. These tube voltages and filter combinations are however uncommon in clinical protocols due to the low photon fluence.

\section{DISCUSSION}

To illustrate the problem with biased $P_{K A}$ reported by built-in KAP meters, a doseoptimisation scenario involving an added copper filter is considered: The automatic exposure control system adjusts the exposure time so that the air kerma at the detector is approximately the same and the decision is made according to displayed $P_{K A}$ values. Most likely, the added filtration will result in lower true $P_{K A}$. But if displayed values for the copper filtration are overestimated by tens of percent, a wrong decision about the usefulness of the copper filter may be made.

IEC $^{(1)}$ requires that the relative combined uncertainty in air kerma-area measurement is less than $\pm 25 \%(\mathrm{k}=2)$. Results in Figure 3(b) show that the vendor implemented a correction for 
additional $\mathrm{Cu}$ filters as the values for $70 \mathrm{kV}$ but different added $\mathrm{Cu}$ thicknesses are close to each other. It is not known to us how the correction is made. The low energy dependence of the relative error $\delta$ in Figure $3 \mathrm{~b}$ suggests that either the data were corrected for energy dependence or a KAP meter with low energy dependence was used.

In equation (4), the relative uncertainty $(\mathrm{k}=2)$ of the calibration coefficient $N_{P_{K A}, Q_{0}}^{r e f}$ of the reference KAP meter was estimated to $\pm 2.4 \%(\mathrm{k}=2)$ by SSM, the uncertainty of its reading $M_{Q}^{\text {ref }}$ was less than $1.0 \%$, the uncertainty of $k_{Q, Q_{1}}^{\text {exp }}$ was about $2.0 \%$ and the uncertainty of $k_{Q_{1}, Q_{0}}^{m o d}$ was less than $4 \%$. In case of $k_{Q, Q_{1}}^{\text {exp }}$, the uncertainty of $0.6 \%$ in the calibration coefficient of the Exradin A3 chamber at RQR5 provided by SSM and the energy dependence less than $1 \%$ were used. The uncertainty of $k_{Q_{1}, Q_{0}}^{\text {mod }}$ is a qualified guess only, the true value is most likely lower for similar beam qualities $Q_{0}$ and $Q_{1}$. A thorough uncertainty analysis of $k_{Q, Q_{0}}^{\text {mod }}$ requires that the errors associated with the computational model are estimated which requires an indepth analysis of how the model parameters influence the beam quality correction factors, including variations in KAP meter design (in particular variations in indium oxide layer thickness), x-ray spectrum (estimation of inherent filtration in clinical beams) and irradiation geometry (scatter and attenuation) which is beyond the scope of this work. Until the uncertainty of the computational method is fully explored, the uncertainty in the combined method is easier to assess and more practicable since the $k_{Q, Q_{1}}^{\exp }$ can be derived experimentally by the medical physicist. The combined relative standard uncertainty of $P_{K A, Q}$ is then estimated using the type B evaluation (chapters 4.3 and 5.1 in $\mathrm{JCGM}^{(11)}$ ) to $5.2 \%(\mathrm{k}=2)$ in compliance with the IAEA ${ }^{(2)}$ recommendation that uncertainty in reported $P_{K A}$ be registered with an accuracy of better than $7 \%(\mathrm{k}=2)$. 
We argue that the reference beam qualities at the standards laboratory and at the clinic should be the same, i.e., $Q_{0}=Q_{1}$, to minimize the uncertainties associated with the transfer of the calibration coefficient from the standards laboratory to the clinic. Experiments with SpekCalc ${ }^{(8)}$ showed that lightly filtered beams were notably affected by uncertainties associated with the limited knowledge about the inherent filtration of the clinical x-ray tube. Heavily filtered beams, on the other hand, resulted in low detector signals and thus increased uncertainties. For these reasons we speculate that reference beam qualities like RQA5 $(70 \mathrm{kV}$, $\mathrm{HVL}=6.79 \mathrm{~mm} \mathrm{Al})$ may be better than RQR5 $(70 \mathrm{kV}, \mathrm{HVL}=2.54 \mathrm{~mm} \mathrm{Al})$ for the heavily filtered clinical beams. Clearly, the best solution would be to implement some heavily filtered beam qualities at the primary and secondary standards laboratories.

The most straightforward and accurate solution for unbiased $P_{K A}$ is that vendors provide beam quality correction factors for their imaging system that will enable the $P_{K A}$ to be measured with an uncertainty better than $7 \%(\mathrm{k}=2)$ in accordance with international recommendations. If vendors cannot provide these factors, then they have to be determined by the medical physicist in order to achieve unbiased $P_{K A}$. These should ideally be applied to the reading of the built-in KAP meter before the total $P_{K A}$ for all exposures in the examination is registered in the Radiological Information System (RIS) repository. Currently, however, the medical physicist often needs to apply one beam quality correction factor after an examination involving several different beam qualities is completed. This may reduce the accuracy of the estimated $P_{K A}$. In this respect an accredited KAP meter function that computes the $P_{K A}$ from knowledge of generator output, added filtration and collimator settings rather than a direct measurement, may be more appropriate, but will require active input from the vendors beyond the requirements of the $\operatorname{IEC}^{(1)}$. Methods to compare the computed $P_{K A}$ with the true $P_{K A}$ are still needed. 
The determination of beam quality correction factors of the built-in KAP meter can be simplified by using an energy independent reference KAP meter. Currently, there are no such KAP meters on the market. The PDC from RadCal (Monrovia, USA) has a suppressed energy dependence $^{(12)}$, but this suppression is not sufficient for measurements aiming at better than $7 \%$ accuracy $(\mathrm{k}=2)$ when only one calibration coefficient is used. Toroi et al ${ }^{(12)}$ showed that the uncertainty of the built-in KAP meter calibration can be less than $5 \%$ when the PDC is used with calibration coefficients depending on the HVL and the beam filtration is less than $0.2 \mathrm{~mm} \mathrm{Cu}$. However, no data were provided for heavily copper-filtered beams. The manufacturer has not disclosed information needed in the computational model of this KAP meter, so the beam quality correction factors cannot be reliably calculated.

A correction for air temperature and air pressure should be applied on readings of vented ion chambers, for instance built-in and reference KAP meters and energy independent ion chambers measuring the air kerma. As the beam quality correction factors typically employ ratios of these quantities, the correction for air temperature and pressure cancels out.

\section{CONCLUSION}

Clinical KAP meters have shown to be biased and correction factors are needed to show the true $P_{K A}$. This is particularly important if $P_{K A}$ is used for optimisation. Vendors are therefore encouraged to provide energy-dependent calibration coefficients for their built-in KAP meters. The less favourable solution is that medical physicists determine the energydependent calibration coefficients by themselves. In this case suitable equipment such as a calibrated reference KAP meter and an energy independent dosemeter will be needed. The results indicated that careful use of the calibration methods presented in this work could 
provide $P_{K A}$ with an uncertainty better than $7 \%(\mathrm{k}=2)$ in compliance with recommendations by IAEA and ICRU.

\section{FUNDING}

This work was funded by the Swedish Radiation Safety Authority, SSM (grant number SSM 2014-1204).

\section{ACKNOWLEDGEMENTS}

This work has been conducted within the Centre for Medical Image Science and Visualization (CMIV) at Linköping University, Sweden. The Department of Radiology at Linköping University hospital is acknowledged for giving access to their x-ray units during daytime. JanErik Grindborg and Jan Lillhök at SSM are acknowledged for providing valuable feedback on this work.

\section{REFERENCES}

1. IEC. Dose Area Product Meters, IEC-60580 (Geneva: International Electrotechnical Commission, 2000)

2. IAEA Dosimetry in Diagnostic Radiology: An International Code of Practice. (TRS No 457) (Vienna: International Atomic Energy Agency, 2007)

3. ICRU. Patient Dosimetry for X Rays Used in Medical Imaging (Report 74). J. ICRU (International Commission on Radiation Units \& Measurements, 2005) (doi:10.1093/jicru/ndi018) 
4. Toroi, P., Komppa, T., and Kosunen, A. A Tandem Calibration Method for Kermaarea Product Meters. Phys. Med. Biol. 53, 4941-4958 (2008) doi:10.1088/0031$9155 / 53 / 18 / 006$.

5. Malusek, A. and Alm Carlsson, G. Analysis of the tandem calibration method for kerma area meters with Monte Carlo simulations. Proceedings of an International Dosimetry Symposium on Standards, Applications and Quality Assurance in Medical Radiation Dosimetry (IDOS) held in Vienna, Austria 9-12 November 2010. 2 Volumes, ISSN 0074-1884, Vol. 1, p. 129-136, ISBN: 978-92-0-116210-6 (International Atomic Energy Agency, 2011)

6. NRPB, National protocol for patient dose measurements in diagnostic radiology. National Radiological Protection Board, Chilton (1992). Available at: https://www.gov.uk/government/uploads/system/uploads/attachment_data/file/337175/ National_Protocol_for_Patient_Dose_Measurements_in_Diagnostic_Radiology_for_w ebsite.pdf (30 October 2015, date last accessed)

7. Malusek, A., Helmrot, E., Sandborg, M., Grindborg J.-E. and Alm Carlsson, G. In-situ calibration of clinical built-in KAP meters with traceability to a primary standard using a reference KAP meter. Phys. Med. Biol. 59, 7195-7210 (2014)

8. Poludniowski, G., Landry, G., DeBlois, F., Evans, P. M. and Verhaegen, F. SpekCalc: a program to calculate photon spectra from tungsten anode x-ray tubes. Phys. Med. Biol. 54, N433-N438. (2009)

9. Salvat F. and Fernández-Varea, J. M. Overview of Physical Interaction Models for Photon and Electron Transport Used in Monte Carlo Codes. Metrologia 46(2): S112S138. (2009)

10. Siemens Product description: Artis zee multi-purpose - Future Version, 2013-11-18 
11. JCGM. Evaluation of measurement data — Guide to the expression of uncertainty in measurement (No. JCGM 100:2008). Joint Committee for Guides in Metrology. (2008)

12. Toroi, P. and Kosunen, A. The energy dependence of the response of a patient dose calibrator. Phys med Biol 54, N151-N156 (2009) 